\title{
Neutron Anisotropy and X-Ray Production of the FN-II Dense Plasma Focus Device
}

\author{
F. Castillo, J.J.E. Herrera, J. Rangel, A. Alfaro, M.A. Maza, V. Sakaguchi, \\ Instituto de Ciencias Nucleares \\ Universidad Nacional Autónoma de México \\ A.P.70-543, Ciudad Universitaria, 04511 México, D.F. México \\ G. Espinosa, and J.I.Golzarri \\ Instituto de Física \\ Universidad Nacional Autónoma de México \\ A.P. 20-364, 01000 México, D.F. México
}

Received on 26 June, 2001

\begin{abstract}
The FN-II Dense Plasma Focus is a small $(<5 k J)$ Mather type device, where the dependence of neutron yield and its anisotropy, in terms of deuterium filling pressure, and the neutron emission angular distribution have been studied. Two different electrode configurations have been tested, showing that their geometry plays an important role both on neutron yield and anisotropy. Time integrated anisotropy has been measured with silver activation counters, on a shot to shot basis. CR-39 nuclear track detectors are used to determine the angular distribution of neutrons, averaged over tens of shots, showing that an isotropic pedestal accounts for $70 \%$ of the emission, while the anisotropy component accounts for the remaining 30\%. The neutron yield shows a tendency to increase with anisotropy, as well as with the emission of hard X-rays observed on-axis. Scintillatorphotomultiplier detectors show a slight Doppler shift in the neutron energy at backward angles, supporting the beam-target mechanism. Additional information has been obtained from time integrated X-ray diagnostics, which include filtered multi-pin-hole cameras.
\end{abstract}

\section{Introduction}

The Dense Plasma Focus (DPF) has been studied since the late 1950's, and its research has been stimulated from its early days because of the simplicity of its engineering, and its properties as an intense, pulsed, low cost, source of neutrons, electron and ion beams, as well as of soft and hard x-rays [1-3]. Although some projections maintain the DPF might still be considered a feasible candidate for a controlled fusion reactor [2,4], most of the recent work has been motivated by ongoing interest as a cost effective pulsed neutron and x-ray source for a wide range of applications [5-9].

The DPF is a coaxial gun, in which the inner electrode, the anode, is electrically insulated from the outer electrode, the cathode, by ceramics or Pyrex. After achieving a high vacuum, gas is admitted at a pressure of a few torr. The plasma originates when a capacitor bank is discharged through a low inductance transmission line, as spark or rail gap switches are closed within a few $n s$ rise time. As the discharge breaks down at the breech of the gun, through the insulator surface, a radial current sheath develops, giving rise to an azimuthal magnetic field, which drives the sheath towards the muzzle. As a result, the sheath sweeps and ionises the neutral gas it finds on its way. Finally, it focuses into a plasma column at the tip of the inner electrode, with densities in the range of $10^{18}-10^{20} \mathrm{~cm}^{-1}$, and electron temperatures in the range of $0.1-2.0 \mathrm{keV}$, depending on the dimensions of the device and the energy stored in the capacitor bank. Confinement is achieved by the pinch effect, due to the axial current, which ranges from $200 \mathrm{kA}$ in small devices up to $2 M A$ in the largest ones. During this phase, soft $\mathrm{X}$ rays are produced by bremsstrahlung. The filling gas can be chosen in order to produce strong line radiation, and thus hard X-rays [10]. The tip of the electrode can be designed in such a way that the impinging electron beam can enhance them.

It is well known from the early days of fusion research that the z-pinch is a magnetohydrodinamically unstable configuration, and that the plasma column is 
destroyed, usually within $10-50 \mathrm{~ns}$. In the process of such instabilities, ions are accelerated away from the anode, while electrons are accelerated into it, associated with the appearence of hot-spots [11]. A reasonable hypothesis is that strong electric fields are generated, which accelerate ions up to energies greater than $10 \mathrm{MeV}$, even in small devices [12-15]. Unfortuantely, discrepancies between conflicting theories have not been solved yet by experimental evidence. Such issue of outmost importance falls beyond the scope of the present paper. If the filling gas is deuterium in a DPF, the accelerated ions react against the background hot dense plasma ions, originating a notorious neutron pulse that ranges from $10^{6}$ per shot in small devices, up to $10^{12}$ in some of the largest ones. This neutron yield follows a power law with current $I$ that goes as $I^{4}$ [16], and with the stored energy $W_{0}$ that goes as $W_{0}^{2}$ [2]. Further studies on the scaling laws of DPF machines have been carried out by Lee and Serban [17].

Throughout the past forty years, the operation of plasma foci has been documented for stored energies that range from $2 k J[6,10,18,19]$ up to $1 M J[2,20]$. The behaviour of small devices around $2 k J$ has been thoroughly studied. The influence of electrode and insulator materials on the neutron emission, has been studied by Rout et al. [18], and comparative studies of ion, x-ray and neutron emission have been carried out [19]. Small portable machines have been proposed for introspective imaging of metallic objects and for neutronic detection of water $[8,9]$. Furthermore, smaller devices with high repetition rate have been developed for applications such as microelectronics lithography [6] and biomedical studies [7]. It can be stated that plasma focus research has spread because of its interesting physics phentomena, its potential applications, the simplicity of its engineering, and its affordability.

The present work stems from the Fuego Nuevo II Dense Plasma Focus Device with two different oxygenfree copper electrodes. The outer electrode is arrayed as a squirrel cage. One of the objectives of this work is to compare the performance of two different inner electrodes with equal length but different diameter. The first electrode, hereafter called electrode I, is a solid cylinder fully contained within the insulator, so its diameter is smaller than the inner diameter of the insulator. The diameter of the second one, hereafter called electrode II, by contrast, matches the outer diameter of the insulator. It may be surmised that the second one may allow a better breakdown, not only because its path is decreased, but also because there is an edge effect at the electrode-insulator joint. It will be shown this is the case.

The structure of the paper is as follows: A brief description of the FN-II device and the diagnostics relevant to this work is made in section II. The latter include time integrated silver activation and nuclear track detectors, which were used to obtain anisotropy and angular distribution measurements, scintillatorphotomultiplier systems, used for time resolved detection of hard X-rays and neutrons, and the multi-pinhole camera. The results for time integrated neutron yield and anisotropy for each electrode will be presented and discussed in section III. Time resolved hard $\mathrm{X}$-rays and neutron signals will be presented in section IV, showing that a slight Doppler effect for the neutron energy can be detected at backward angles. Time integrated pin-hole x-ray signals, and radiographs obtained on axis will be shown in section V. Finally, some conclusions will be drawn in section VI.

\section{Description of the experimen- tal device}

A scheme of the experimental arrangement is shown in Fig. 1. Two different inner electrodes (anodes) of equal length (40 $\mathrm{mm}$ long), but differing diameter have been tested. The insulator used in both cases is a $10 \mathrm{~mm}$ long Pyrex sleeve, with an outer diameter of $50 \mathrm{~mm}$. The diameter of electrode I is $40 \mathrm{~mm}$, while that of electrode II matches the outer diameter of the Pyrex sleeve. The squirrel cage outer electrode (cathode) consists of 12 copper bars, of $8 \mathrm{~mm}$ diameter and $36 \mathrm{~mm}$ long each, arrayed on a $100 \mathrm{~mm}$ diameter circle. The capacitor bank consists of four $1.863 \mu F$ capacitors in parallel, with an internal inductance of $24 \mathrm{nH}$ each. The energy is conducted through a parallel copper plate transmission line insulated by six Mylar sheets, with a total thickness of $2.2 \mathrm{~mm}$. The inductance of the system has been measured by short-circuiting the gun, and found to be $54 n H$. The circuit is closed by a spark gap switch, pressurised with air, and triggered by a micro Marx generator, which produces a $100 \mathrm{kV}$ pulse with a $20 \mathrm{~ns}$ rise time. The results obtained in this work were produced by charging the capacitor bank at $36 k \mathrm{~V}$, which means the stored energy was $4.6 \mathrm{~kJ}$. 


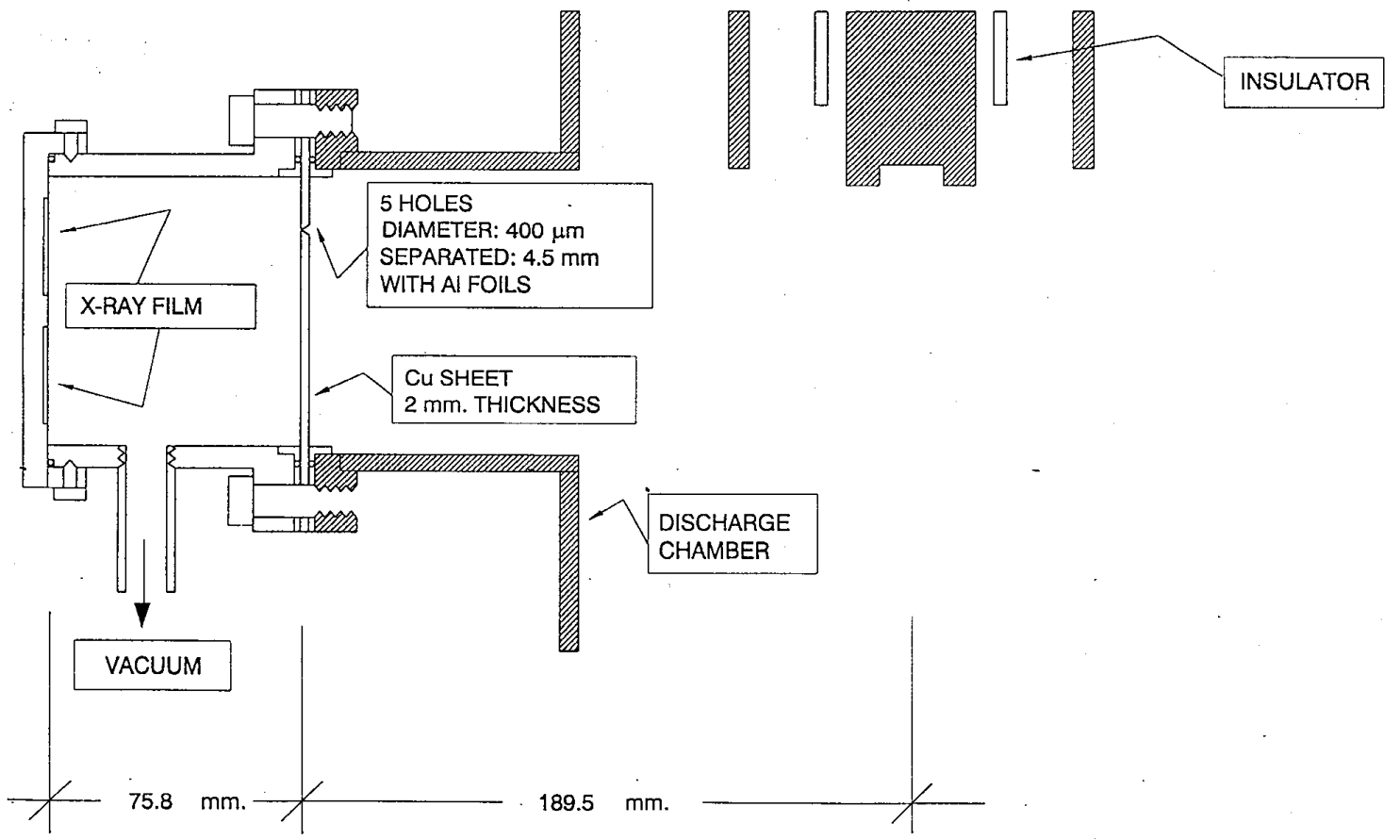

Figure 1. Scheme of the experimental arrangement, showing electrode I and the pin-hole camera.

The behaviour of the circuit is monitored with a Rogowski coil which yields the derivative of the current signal $d I / d t$. Fig. 2 shows a typical oscillogram, where (a) is the $d I / d t$ signal, and (b) is the signal from a scintillator-photomultiplier detector, described below. The dip near $d I / d t=0$, corresponds to a sudden drop of current, which occurs as impedance rises during the compression of the plasma column. This is the signature of the focusing of the plasma column.

Time integrated measurements of the neutron yield and its anisotropy are made with two different methods. Silver activation detectors are used on a shot to shot basis. These consist in two Victoreen 1B85 Geiger-Muller counters, with lateral window, $10 \mathrm{~cm}$ long, wrapped up in a $500 \mu \mathrm{m}$ silver foil, and surrounded by $6 \mathrm{~cm}$ of paraffin. The total size of each detector is $22 \mathrm{~cm}$ high with a $15.5 \mathrm{~cm}$ diameter. Their purpose is to measure the $\beta$ decay of ${ }^{108} \mathrm{Ag}$ and ${ }^{110} \mathrm{Ag}$ nuclei that result from the activation of ${ }^{107} \mathrm{Ag}$ and ${ }^{109} \mathrm{Ag}$, respectively. The paraffin moderates the neutrons, therefore increasing the activation cross section. Both counters were placed $54 \mathrm{~cm}$ away from the focus, one of them on the axis of the device, and the other one at $90^{\circ}$. They were calibrated with an Am-Be neutron source, and the background counts were monitored on a daily basis, in order to check the invariance of their behaviour. According

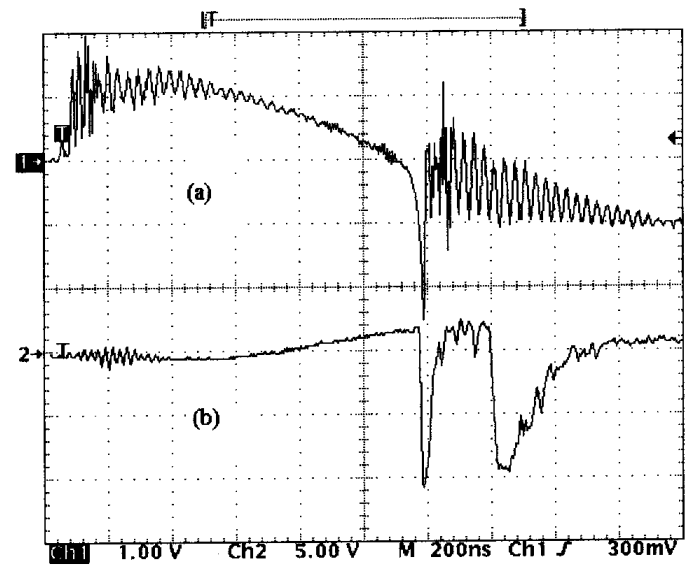

Figure 2. A typical oscillogram showing the $d I / d t$ signal (a) along with the one of the NE211 scintillator-photomultiplier detector placed $5.4 \mathrm{~m}$ away from the column at $90^{\circ}(\mathrm{b})$. The dip in signal (a) is the signature of the pinch at maximum current. The first pulse in (b), which almost coincides with the dip, corresponds to hard X-rays, and the second one to neutron emission.

to the calibration, the behaviour of both counters was very similar, and could be considered to be the same within two significant figures. The neutron yield per steradian, measured by each counter, is the number of 
counts collected in $60 \mathrm{sec}$ multiplied by $(9.4 \pm 0.3) \times 10^{3}$. The minimum neutron yield threshold per steradian per minute that can be measured, determined by the background, is $(4.7 \pm .9) \times 10^{4}$. Further information on this method can be found in Ref.[21].

The second method used to measure the neutron anisotropy, and specifically the average neutron angular distribution, consisted in using CR-39 plastic nuclear track detectors. Fast neutrons can be detected with the CR-39 polycarbonate $\left(\mathrm{C}_{12} \mathrm{H}_{18} \mathrm{O}_{7}\right)$, either by elastic scattering with protons from the detector material or radiator foils, such as polyethylene placed in front [22], or by $\alpha$ particles produced by ${ }^{10} B(n, \alpha)$ reactions [23]. At higher energies, even scattered $C$ and $O$ atoms generate etchable tracks [22]. These tracks can be chemically etched by agents such as $\mathrm{NaOH}$ or $\mathrm{KOH}$. This method has been used earlier at other devices to detect neutrons of energy around $2.45 \mathrm{MeV}$ [24]. However it was not used to evaluate the role of the angular distribution in the determination of the total neutron yield, which is one of the purposes of this work. Rectangular chips, with a $1.9 \mathrm{~cm} \times 0.9 \mathrm{~cm}$ surface and $500 \mu \mathrm{m}$ thickness were placed on a circular arc of 100 $\mathrm{cm}$ radius, centred at the focus, outside the discharge chamber. Five different angles relative to the gun axis were chosen: $0^{\circ}, 45^{\circ}, \pm 90^{\circ}, 135^{\circ}$ and $170^{\circ}$. It was found that, in order to detect neutrons, the CR-39 needed to be covered with polyethylene sheets, $2 \mathrm{~mm}$ thick. The detectors were exposed to 65 shots, for a filling pressure of 2.75 torr. This particular choice of pressure will be explained below. The chemical etching of the plastic chips was performed by immersing them in $\mathrm{KOH}$, $6.25 \mathrm{M}$ solution, at $(60 \pm 1)^{\circ} \mathrm{C}$ for 6 hours. The track counting was done with an optical microscope, with a $200 \times$ magnification, fixing a field of $0.36 \mathrm{~mm} \times 0.3 \mathrm{~mm}$ as measurement area. Forty fields per chip were counted, in each chip, in order to obtain better statistics. The optical microscope is coupled to a CCD camera and a Digital Image Analysis System (DIAS) [25], making all the measurements process automatically.

Two scintillator-photomultiplier detectors have been used for time resolved neutron detection. The scintillators, NE211 and NE218, are both contained within cylinders of equal size; $7 \mathrm{~cm}$ long and $5 \mathrm{~cm}$ diameter. They are coupled to AMPEREX photomultiplier tubes model 56 AVP. In order to minimise the hard X-ray signal, the scintillators are surrounded by a $1 \mathrm{~cm}$ lead shield, and their front is covered by lead and copper filters. Signal (b) in Fig. 2 was obtained with the NE211 scintillator and a Pb filter $5.53 \mathrm{~mm}$ thick, followed by a $2.65 \mathrm{~mm} \mathrm{Cu}$ foil. The peak that almost coincides with the dip of the Rogowski coil signal is produced by hard X-rays, and it is clearly resolved from the neutron pulse by time of flight. Thus, if the energy of the neutrons varies with the angle, this can be determined as a Doppler shift, measuring the difference in the time of flight between the X-ray and the neutron peaks at different angles. Further details will be given in section $\mathrm{V}$.

Although the inner electrode is a solid cylinder, the centre of the tip is hollow, so that hard X-rays from the collision of the electron beam with it are hidden from direct observation at $90^{\circ}$ from the axis. A multi-pin-hole camera was placed in that direction, for time integrated $\mathrm{X}$-ray imaging of the plasma column. Five circular pinholes, $400 \mu \mathrm{m}$ in diameter, and separated by $4.5 \mathrm{~mm}$ were made on a $2 \mathrm{~mm}$ thick $\mathrm{Cu}$ screen, $19 \mathrm{~cm}$ away from the axis. Each hole was covered by 25,10,5,15 and $20 \mu m$ thick Al filters respectively. Although the $400 \mu m$ diameter of the pinholes may be too large for getting a proper resolution, it was chosen in order to guarantee reproducibility of the five holes. The images were recorded on X-ray dental film, placed within an evacuated camera $7.6 \mathrm{~cm}$ away from the copper screen, on a revolver-like film back with capacity for four films. This yields a .40 magnification of the observed object. The time (3 min.) and temperature $\left(20^{\circ} \mathrm{C}\right)$ of the developing process were controlled, so the intensities obtained for different shots could be compared.

Besides the pin-hole camera, a $300 \mu \mathrm{m} \mathrm{Al}$ window was placed on axis, $35 \mathrm{~cm}$ away from the inner electrode, which allows observation of the hard X-rays produced on the face of the inner electrode.

\section{Neutron yield and anisotropy results}

Two silver activation detectors, as described above, were used to measure the neutron yield and anisotropy for electrodes I and II. The number of counts obtained during sixty seconds after each shot, were recorded for one detector placed on axis $\left(0^{\circ}\right)$ and the other at $90^{\circ}$. A wide range of filling pressures was explored, going from 1.0 to 4.5 torr. The average number of counts $N_{0}$ for the detector on axis, and $N_{90}$ for the detector at $90^{\circ}$, as a function of filling pressure are shown in Fig. 3(a) for electrode I and Fig. 3(b) for electrode II. The case of electrode I includes 400 shots, while that of electrode II includes 539 shots. It must be noted that for electrode I the number of counts is barely above the background for pressures below 1.5 torr, while electrode II showed a larger neutron yield at low pressures. It is also observed that the error bars, which show the mean square deviation, are smaller for electrode II. This reflects a better reproducibility for this geometry. Generally speaking, the neutron yield is clearly larger for electrode II than for electrode I, the maximum being roughly 3 times as large for the first one. This maximum appears around 3.50 torr for electrode I, and is shifted down to 2.75torr for electrode II. The corresponding scale for $d Y_{N} / d \Omega$ is given on the right of Figs. 3(a) and (b). 

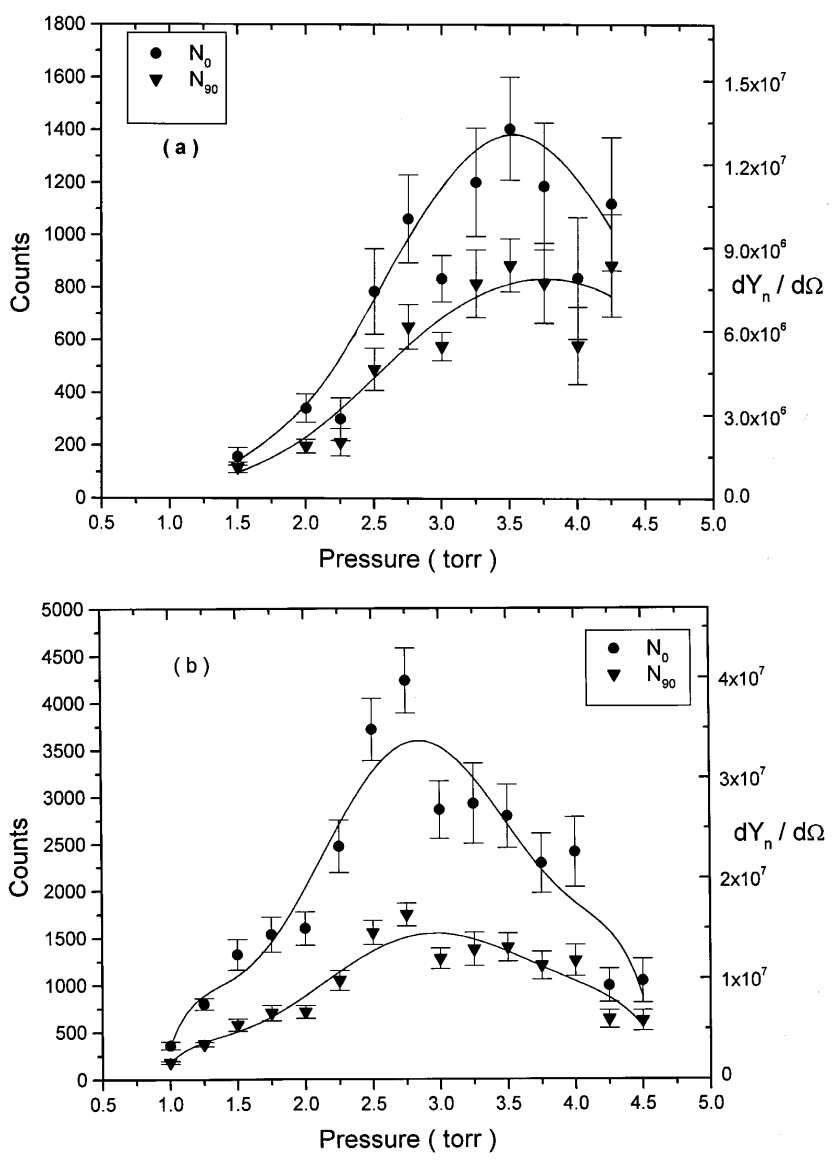

Figure 3. Number of counts obtained during one minute after each shot with the silver activation detectors at $0^{\circ},\left(N_{0}\right)$ and $90^{\circ},\left(N_{90}\right)$, as functions of the deuterium filling pressure. Results for electrode I are shown in (a), while those for electrode II are shown in (b). The right hand scale shows the diferential yield per solid angle.

The data for both electrodes show a clearly defined anisotropy $A=N_{0} / N_{90}$, which is shown as a function of the deuterium filling pressure in Fig. 4. Electrode II shows a stronger $A$, which tends to be larger for lower pressures, up to 2.75torr, and falls thereafter. The anisotropy for electrode I generally falls below that of electrode II, and remains roughly constant, within the error bars, throughout the range of pressures explored. A better reproducibility is found for electrode II again. It may also be interesting to look at the scattergrams of $A$ for several shots vs. the number of counts of the detectors. As a best case sample, Fig. 5 shows $A$ vs. $N_{0}$ for electrode II. A linear regression shows a clear tendency of anisotropy to grow with the number of counts. This result shows the relevance of the beam-target effect as a neutron generation mechanism. It may be surmised that the better the ion beam generation, the larger the neutron yield will be for a given stored energy.

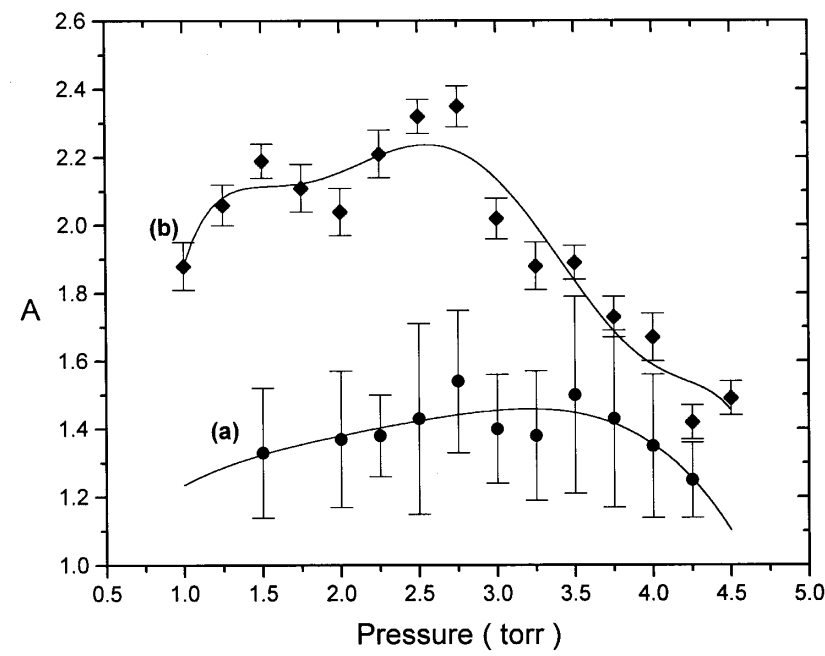

Figure 4. Time integrated anisotropy for electrodes I (a) and II (b) as a function of the deuterium filling pressure.

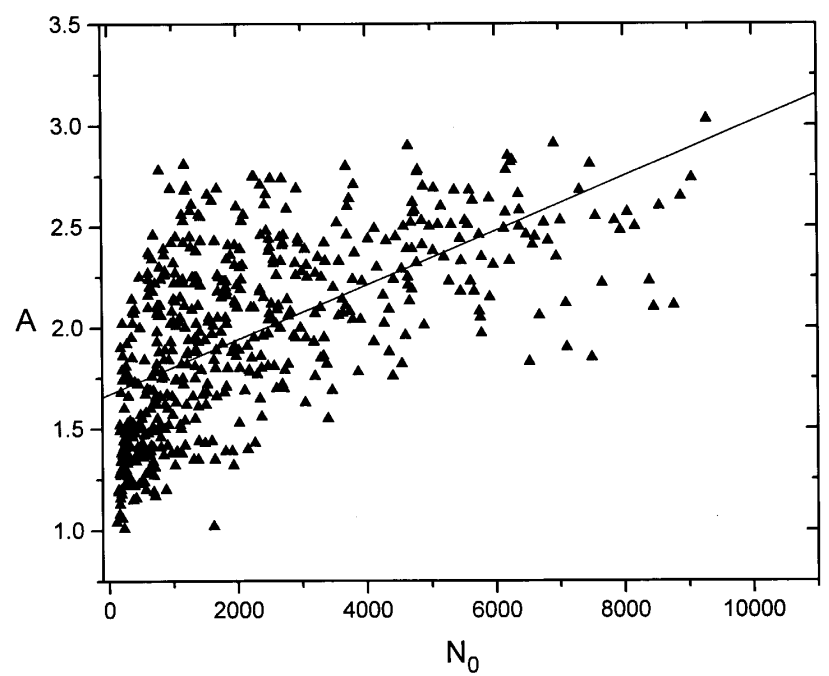

Figure 5. Scattergram of anisotrpy $A$ vs. the number of counts on axis $N_{0}$ for electrode II.

Once the optimum pressure 2.75torr was found for electrode II, the average angular distribution of the neutron yield was determined using the CR-39 detectors. The angular distribution of the track density, obtained with 65 shots is shown in Fig. 6, normalised to the average value at $0^{\circ}$. The data obtained for positive angles can be adjusted by a Gaussian function superposed on a pedestal;

$$
f(\theta)=B+\frac{C}{\sigma \sqrt{2 \pi}} \exp \left(-\theta^{2} / 2 \sigma^{2}\right),
$$

where $\theta$ is the angle in degrees, $B=.507, C=82.7$ and $\sigma=70.6$. The values obtained for the samples at $\pm 90^{\circ}$ confirm the symmetry of the distribution, as can be seen from the figure. Eq.(1) can be used in order to estimate by integration the total neutron yield, introducing a proper scaling factor for $B$ and $C$, using the silver activation counters data. The isotropic pedestal contributes $70 \%$ of the neutron yield, while 
the anisotropic Gaussian distribution accounts for the remainig $30 \%$. For the case of the 65 shots, for which Fig. 6 was obtained, the average number of counts on axis and at $90^{\circ}$ are $\left\langle N_{0}\right\rangle=2034$ and $\left\langle N_{90}\right\rangle=1265$, which yield $d Y_{0} / d \Omega=1.9 \times 10^{7}$ and $d Y_{90} / d \Omega=1.2 \times 10^{7}$ neutrons/str, respectively. From these values, and the ones obtained from the adjusted curve, eq.(1), for both positions, an optimistic scaling factor $S_{1}=2.0 \times 10^{7}$ is obtained at $0^{\circ}$, and a pesimistic one $S_{2}=1.7 \times 10^{7}$ at $90^{\circ}$. They agree within $18 \%$. Integrating eq.(1) over the sphere, and taking the average between the values obtained for $S_{1}$ and $S_{2}$, the total neutron yield per shot, for this particular set of 65 shots is found to be $Y_{N}=(1.66 \pm 0.14) \times 10^{8}$. This value is $11 \%$ higher than the one expected from the measurements at $90^{\circ}$, which would be $1.5 \times 10^{8}$, assuming the emission were isotropic.

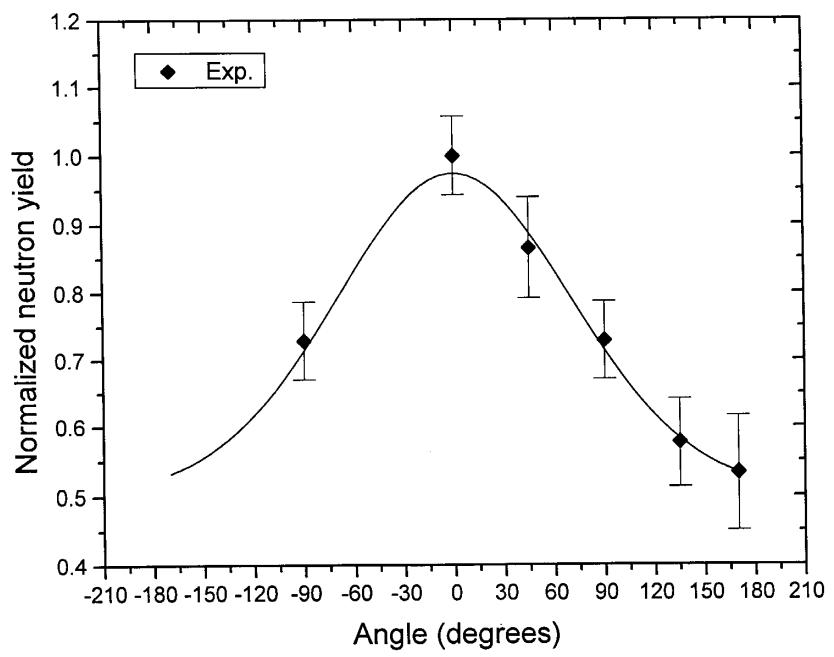

Figure 6. Angular distribution of the track population obtained from the neutron emission, normalised to the maximum count obtained for $0^{\circ}$. CR-39 chips covering a $1.9 \mathrm{~cm} \times 0.9 \mathrm{~cm}$ area were placed $1 \mathrm{~m}$ away from the focus at $0^{\circ}, 45^{\circ}, \pm 90^{\circ}, 135^{\circ}$ and $170^{\circ}$ from the axis.

The value of $A$ obtained from the CR-39 data is $1.37 \pm .05$. For this particular set of shots, the anisotropy measured with the silver activation detectors, obtained on a shot to shot basis, is $1.55 \pm 0.22$, which is consistent within the experimental error. Besides counting the track density by means of DIAS, their diameters were also measured. All five chips were considered for this measurement. More than $90 \%$ of the tracks fall within a strongly peaked Gaussian distribution centred around $19.8 \mathrm{~mm}$, with a full width at half maximum of $4.3 \mathrm{~mm}$. These are presumably produced by protons kicked off the polyethylene plate by elastic $(n, p)$ scattering. This is an indication that the neutrons that are being detected are strongly mono-energetic. The remaining $10 \%$ of the tracks are attributable to a neutron background, which results from reflections on the floor and walls.

\section{Time integrated X-ray re- sults}

Time integrated soft X-ray images of the plasma column have been obtained with a multi-pin-hole camera, as that described in section II. Typical images obtained with electrode I are shown in Figs. 7a and b. In the first case $m=0$ and bright-spots can be observed. The length of the central image is $8 \mathrm{~mm}$, which means the maximum size of the plasma column is around $20 \mathrm{~mm}$. This case corresponds to shot 2578 , where $N_{0}=283$ and $N_{90}=164$. An interesting example is that of shot 2582, shown in Fig. 7b, where the column is kinked, and a hot-spot appears in the kinked column. It looks like an $m=0$ instability occurs well after the plasma column has been bent as a result of an $m=1$ instability. Obviously, this statement cannot be conclusive without the support of time resolved diagnostics. In this case $N_{0}=523$, while $N_{90}=372$. However, these two cases differ significantly from the images obtained with electrode II, which are generally dimmer. A good example is that of Fig. 8a, corresponding to shot 2745, where the neutron yield is considerably larger: $N_{0}=8876$ and $N_{90}=3347$. This hints at the possibility that the radiation from electrode $I$ has a stronger line radiation component originated by impurities, than that of electrode II. Since the neutron yield is clearly greater for the latter, it is apparent that the impurity radiation cooling acts in detriment of neutron production.

Harder X-rays cross the $300 \mu \mathrm{m} \mathrm{Al}$ window on the axis which can be used for radiographical purposes, such as the those shown in Fig. 8, where the inner details of a pencil can be observed. There is an inverse relationship between the intensities of such onaxis X-rays and the soft X-rays at the pin-hole camera. This can be seen as follows: An array of superposed $\mathrm{Pb}$ foils, each $63 \mu \mathrm{m}$ thick was prepared, such that the maximum thickness corresponds to five foils $315 \mu \mathrm{m}$. For shot 2745, shown in Fig. 8a, the radiograph of the $\mathrm{Pb}$ filter obtained on axis is shown in Fig. 8b. In contrast, for shot 2744, where the neutron yield is lower $\left(N_{0}=1329, N_{90}=706\right)$, the soft $\mathrm{X}$-rays at the pinhole camera are stronger, as shown in Fig. 10a, while the radiograph on axis, Fig. 10b, is considerably dimmer than for the former case. This pattern is systematically observed. It may surmised that, since the hard X-rays observed on axis are produced by the electron beam that collides on the inner electrode, the stronger the beam, the greater is the neutron yield. Thus, the electron acceleration mechanism may be correlated with the neutron generation mechanism. Further time resolved studies may clarify this possibility. On the other hand, the pinhole cameras indicate that purer, impurity-free plasmas allow a better neutron yield. This is consistent with the discussion in the previous paragraph. 


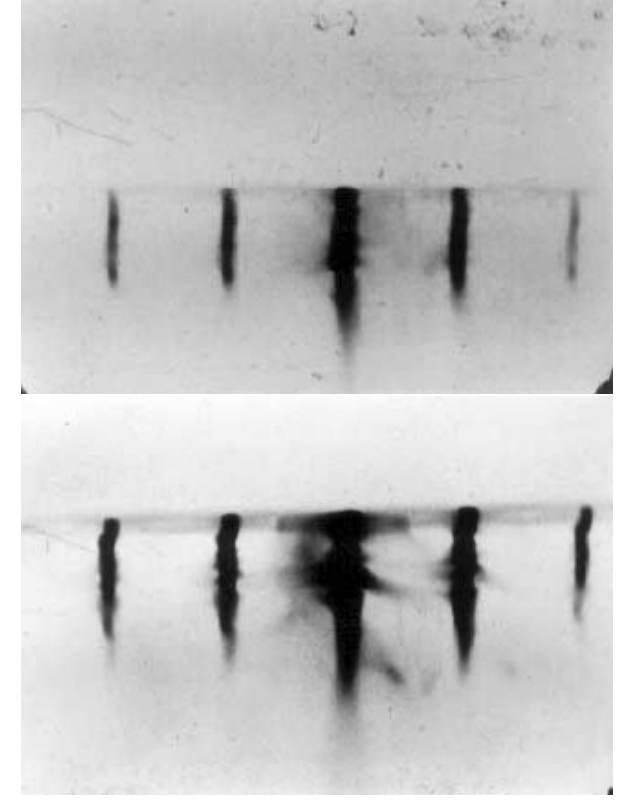

Figure 7. X-ray images for shots 2578 (a) and 2582 (b), obtained with the multi-pin-hole camera shown in Fig. 2. In both cases $m=0$ instabilities can be observed. In shot 2582 the plasma column is kinked,showing an $m=1$ instability, and a hot spot can be observed at the tip.

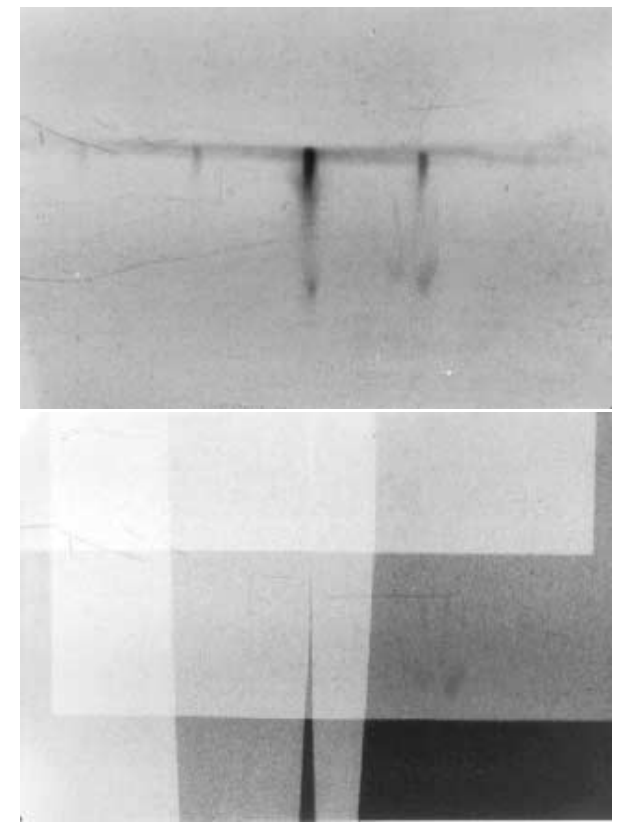

Figure 8. (a) X-ray pin-hole images for shot 2745. The neutron yield increases significantly with electrode II, but the images are dimmer. (b)Radiograph of an array of five $\mathrm{Pb}$ foils, obtained with the X-rays emitted on axis for shot 2745. The maximum thickness, $315 \mu \mathrm{m}$, appears on the upper left corner, while the minimum, $63 \mu \mathrm{m}$, appears on the lower right corner.

\section{Time resolved hard X-ray and neutron measurements}

Two scintillator-photomultiplier systems have been used for hard X-ray and neutron measurements, as described in section II. The geometry is similar for both, but in one case the scintillator used is NE-211, while in the other one it is NE-218. Signal (b) in Fig. 2 was obtained with the NE-211 at $5.4 m$ from the axis of the device. The first peak that almost coincides with the dip of the $d I / d t$ signal is a hard X-ray pulse, related to the electron acceleration in the focus phase, while the neutron pulse is observed in the second peak, retarded by their time of flight. For this particular shot, 2909, $N_{0}=8545$ and $N_{90}=3284$. The two scintillators used in this work have similar response to the neutron signal within $20 \mathrm{~ns}$. In the case of the x-ray signal the difference is indistinguishable.

The NE-211 was found to be more effective. This can be seen in Fig. 11, where both detectors were placed at the same distance of $5.4 \mathrm{~m}$, with $6.4 \mathrm{~mm} \mathrm{~Pb}$ and $1.3 \mathrm{~mm} \mathrm{Cu}$ filters. For this shot, $3461, N_{0}=1039$, and $N_{90}=735$. In the oscilogram shown in Fig. 11, both detectors were placed at $5.4 \mathrm{~m}$ from the focus, but at different angles. The NE218 detector (signal (b)) was placed at $90^{\circ}$ from the axis, while the NE211 detector (signal (a)) was placed at a $170^{\circ}$ backwards angle. The former was filtered with $6.4 \mathrm{~mm} \mathrm{~Pb}$ and $1.3 \mathrm{~mm} \mathrm{Cu}$, and the latter with a set of $5.35 \mathrm{~mm} \mathrm{~Pb}$ and $2.65 \mathrm{~mm}$ $\mathrm{Cu}$. The hard X-ray peak can be used as a time reference for the occurrence of the focus, and the energy of the neutrons can be measured in terms of the difference of the time elapsed between both peaks, the main problem being that neutrons emitted at a later time can overlap neutrons emitted earlier with smaller energy. Thus, for $5.4 \mathrm{~m}$, the equivalence between the time difference and the energy of neutrons is given by $\Delta(t)(\mathrm{sec})=.397 \times 10^{-6} E(\mathrm{MeV})^{-1 / 2}$. From the difference of occurrence of the maxima of both detectors, a Doppler shift in the energy is clearly observed, despite of the experimental error. The results are shown in Table 1.

Table 1. Energy Doppler shifts of neutrons measured at backward angles. The second column gives the time of flight difference that exists between the neutron peak measured by the detector placed perpendicular to the axis, and that measured by the detector at an angle, given by the first column.

\begin{tabular}{|c|c|c|}
\hline $\begin{array}{c}\text { Angle with } \\
\text { respect to axis }\end{array}$ & $\begin{array}{c}\text { Time of flight } \\
\text { difference (nsec) }\end{array}$ & $\begin{array}{c}\text { Energy shift } \\
\text { MeV }\end{array}$ \\
\hline $120^{\circ}$ & $18 \pm 5$ & $0.3 \pm 0.1$ \\
$150^{\circ}$ & $22 \pm 5$ & $0.4 \pm 0.1$ \\
$170^{\circ}$ & $36 \pm 5$ & $0.6 \pm 0.1$ \\
\hline
\end{tabular}




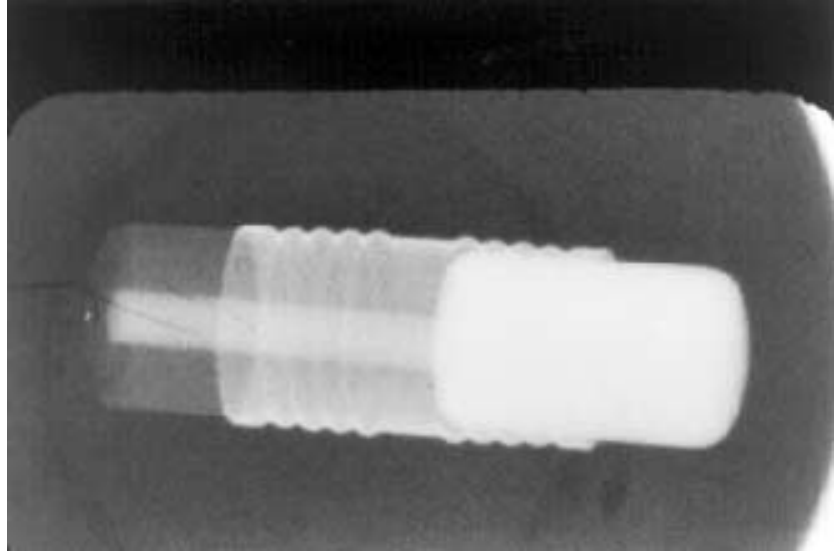

Figure 9. Radiograph of a pencil obtained with the X-rays that cross a $300 \mu \mathrm{m} \mathrm{Al}$ window on axis. Inner details can be clearly observed.
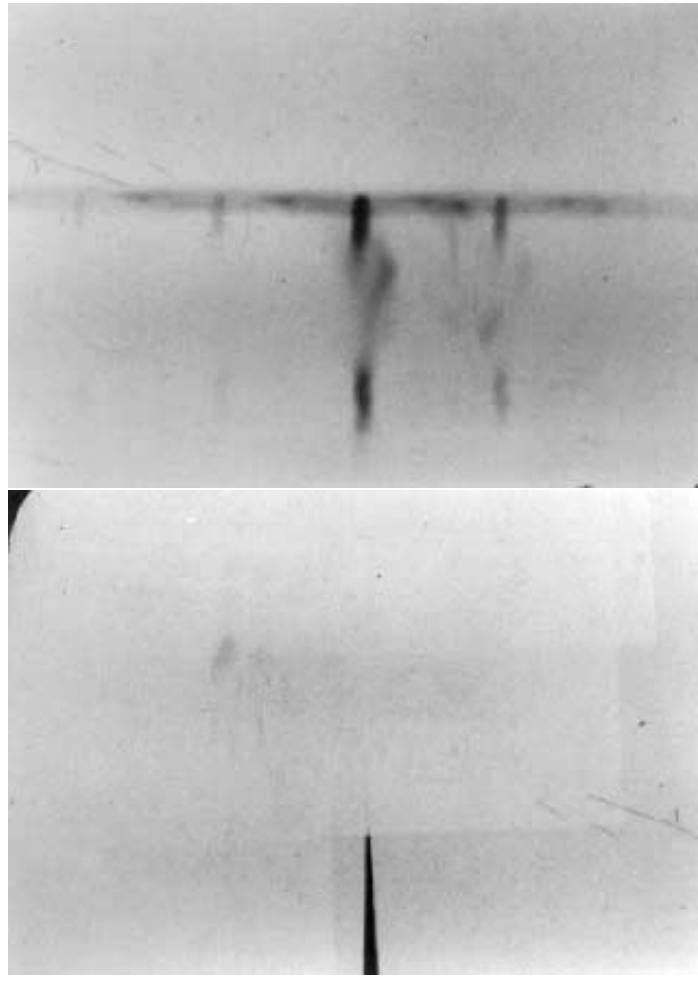

Figure 10. Same as Fig.8, but for shot 2744, which produced a smaller neutron yield: (a) Soft X-ray images obtained with the multi-pin-hole camera. (b) The corresponding radiograph of the $\mathrm{Pb}$ foil array.

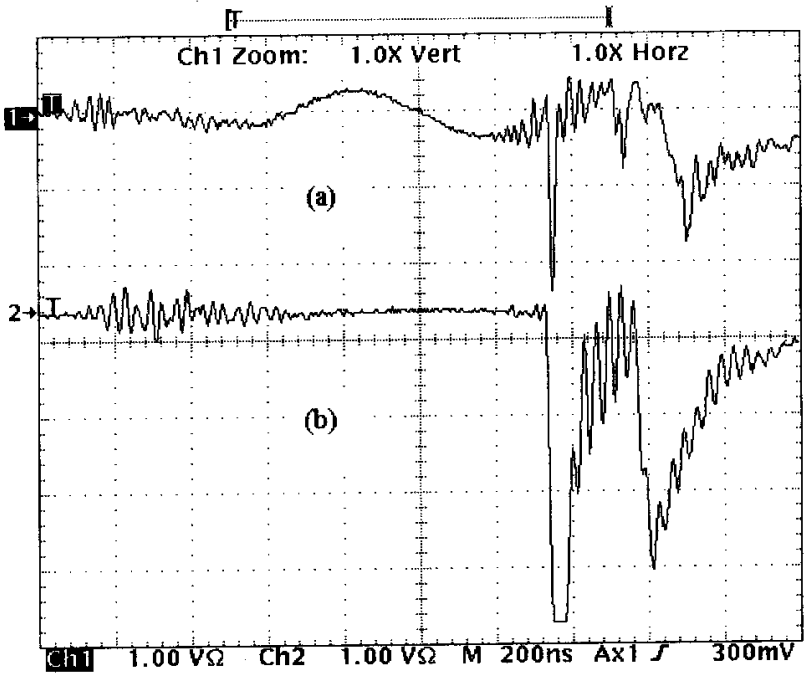

Figure 11. Hard X-ray and neutron signals, obtained for shot 3830 , with the NE211 detector (a) at $5.4 \mathrm{~m}$ from the device and a $170^{\circ}$ backward angle, and the NE218 detector (b) at the same distance, but perpendicular to the axis. A Doppler shift is clearly seen as a difference of the neutron time of flight for each detector.

\section{Conclusions}

Two electrode configurations have been tested: one where the electrode is fully contained within the insulator (electrode I), and one where the electrode diameter matches the one of the insulator (electrode II). The neutron yield and anisotropy was found to be substantially larger for the latter case, while an improvement in reproducibility was also observed. This may be related to a better current sheath formation at the breakdown phase, as a result of both the edge effect of the electrode, close to the insulator, and a decrease in the breakdown length.

This work provides evidence that neutron yield tends to increase with anisotropy in this device, which points to the convinience of the beam-target effect for neutron generation. From the average angular distribution of the neutron emission, measured with CR-39 nuclear track detectors, it is found that there is an isotropic pedestal that accounts for $70 \%$ of the emission, while the anisotropy component accounts for the remaining $30 \%$. The estimation of the total neutron yield using this information is $11 \%$ larger than what would be expected form the silver activation detector measurement at $90^{\circ}$, assuming the emssion were isotropic. Further evidence of the existence of the beam-target effect is demonstrated by a Doppler effect observed at backward angles with scintillator-photomultiplier systems.

The pinhole camera images tend to be dimmer for high neutron yield shots, which may be related to a smaller line radiation from impurities, and consequently lower radiation cooling. These images are generally 
dimmer for electrode II than for electrode I. On the other hand, the fact that a dimmer emission of soft Xrays at $90^{\circ}$, together with a brighter emission of $\mathrm{X}$-rays on axis are observed when the neutron yield is larger, seems to indicate that the neutron generation is related to the formation of the electron beam that impinges on the electrode. It may be interesting to note that after 560 shots, the electron beam drilled an almost cylindrical hole, $3 \mathrm{~mm}$ wide and $6 \mathrm{~mm}$ deep, in electrode I, while the erosion in electrode II, after 956 shots showed as a cone, $6 \mathrm{~mm}$ wide and $12 \mathrm{~mm}$ deep. Both in the cases of soft x-ray emissions at $90^{\circ}$, and hard x-ray emissions on the axis, spectroscopy will be made in future work, in order to clarify the nature of the phenomena.

Regarding the pin-hole $\mathrm{x}$-ray images, it is interesting to point out that, in some cases, bright spots that are related to $m=0$ instabilities are observed in kinked columns which presumably arise from $m=1$ instabilities. This might mean that under certain circumstances the $m=1$ instability may have a faster growth rate than $m=0$ instabilities, against common wisdom. However, study of this phenomena with time resolved optical diagnostics are needed in order to clarify this.

\section{Acknowledgments}

The authors wish to acknowledge the fruitful interaction with Jorge Pouzo. F. Castillo is particularly grateful for his hospitality at the Universidad del Centro de la Provincia de Buenos Aires. This work was partially supported by DGAPA-UNAM project IN105100, and the International Atomic Energy Agency, that made possible the attedance of J.J.E. Herrera to this meeting.

\section{References}

[1] J.M. Mather, "Dense Plasma Focus", in Methods in Experimental Physics, Vol.9, Part B, ed. R.H.Lovberg and H.R.Griem (Academic Press, Nueva York, 1971) pp.187-249.

[2] J.S. Brzosko, J.H. Degnan, N.V. Filippov, B.L. Freeman, G.F. Kiutlu and J.W. Mather, "Comments on the Feasibility of Achieving Scientific Break-even with a Plasma Focus Machine", in Current Trends in International Fusion Research, ed. E.Panarella (Plenum Press, New York, 1997) pp. 11-32.

[3] A. Bernard, H. Bruzzone, P. Choi, H. Chuaqui, V. Gribkov, J. Herrera, K. Hirano, A. Krejci, S. Lee, C. Luo, F. Mezzetti, M. Sadowski, H. Schmidt, K. Ware, C.S. Wong and V. Zoita, J. Moscow Phys. Soc. 893 (1998).

[4] J. Pouzo, "Project of a Major DPF Facility Based on Original Criteria of Design Optimizing the Neutron Yield Performace" in Proc. Intl. Symp. Plasma '97: Research and Applications of Plasmas Vol.1, ed. Sadowski M and Rothkaehl H (Space Research Center PAS, Poland, 1997) pp.65-71.
[5] R. Lebert, A. Engel, K. Bergmann, O. Treichel, C. Gavrilescu and W. Neff, "Compact Plasma Focus Devices: Flexible Laboratory Sources for Applications" in Dense Z-Pinches, Fourth International Conference ed. N. Pereira, J. Davis and P.E. Pulsifer (AIP Conference Proceedings 409, Woodbury, New York, 1997) pp.291298.

[6] S. Lee, P. Lee, G.Zhang, X. Feng, V.A. Gribkov, M. Liu, A. Serban and T.K. Wong IEEE Trans. Plasma Science 26, 1119 (1998).

[7] A.V. Dubrovsky, V.A. Gribkov, T.A. Kozlova, L.V. Volobuev, and M.A. Orlova J. Tech. Phys. 39, Special Suppl. 133 (1998).

[8] C. Moreno, A. Clausse, H. Bruzzone, J. Martínez, R. Llovera, and A. Tartaglione, "Small-Chamber 4.7 kJ Plasma Focus for Applications" in Proc. IX Latin American Workshop on Plasma Physics ed. H. Chuaqui and M. Favre, (AIP Conference Proceedings 563], Woodbury, New York,2001) pp. 276-281.

[9] C. Moreno, A. Clausse, J. Martínez, R. Llovera, A. Tartaglione, M. Vénere, R. Barbuzza and M. del Fresno, "Using a 4.7 kJ Plasma Focus for Introspective Imaging of Metallic Objects and for Neutronic Detection of Water" in Proc. IX Latin American Workshop on Plasma Physics ed. H. Chuaqui and M. Favre (AIP Conference Proceedings 563), Woodbury, New York, 2001) pp. 300-305.

[10] F.N. Beg, I. Ross and A.E. Dangor, "X-ray Emission from a $2 \mathrm{~kJ}$ Plasma Focus" in Dense Z-Pinches, Fourth International Conference, Vancouver, 1997, ed. N. Pereira, J. Davis and P.E. Pulsifer (AIP Conference Proceedings 409, Woodbury, New York, 1997) pp. 339-343.

[11] L. Jakubowski, M. Sadowski and E. Baronova, "Experimental Studies of Hot-Spots Inside PF Discharges with Argon Admixtures" in Proc. 1996 Int. Conf. on Plasma Physics, Nagoya, 1996, ed. H. Sugai and T. Hayashi (Arakawa Printing Co., Japan, 1997) pp. 1326-1329.

[12] M.G. Haines, Nucl. Instrum. Methods 207, 179 (1983).

[13] V.V. Vikhrev, Sov.J.Plasma Phys. 12, 262 (1986).

[14] B.A. Trubnikov, Sov.J.Plasma Phys. 12, 271 (1986).

[15] R. Deutsch and V. Kies, Plasma Phys. and Controlled Fusion 30, 263 (1988).

[16] M. Milanese and J. Pouzo, "Neutron Yield Scaling Laws for Plasma Focus Devices", in Small Plasma Physics Experiments, ed. Lee S and Sakanaka P H (World Scientific, Singapore, 1988) pp.66-79.

[17] S. Lee and A. Serban, IEEE Trans. Plasma Science 24, 1101 (1996).

[18] R.K. Rout, A.B. Garg, A. Shyam, and M. Srinivasan, IEEE Trans. Plasma Science 23, 996 (1995).

[19] M. Zakaullah, I. Akhtar, A. Waheed, K. Alamgir, A.Z. Shah, and G. Murtaza, Plasma Source Sci. Technol. 7, 206 (1998). 
[20] M. Scholz, M. Borowiecki, L. Karpinski, R. Miklaszewski, W. Stepniewski, M. Sadowski, A. Szydlowski, V.M. Romanova, S.A. Pikuz, and T.Ya. Faenov, "X-Ray Emission from a Megajoule Plasma-Focus Experiment" in it Proc. Intl. Symp. Plasma '97: Research and Applications of Plasmas Vol.2, ed. Sadowski M and Rothkaehl H (Space Research Center PAS, Poland, 1997) pp.125-128.

[21] A. Gentilini, J.P. Rager, M. Tacchi, D. Antonini, B. Arcipiani, E. Moioli, E. Pedretti, and R. Scafe, Nucl. Instrum. Methods 172, 541 (1980).
[22] H.J. Brede, A. Gisbertz, I. Kohler, E. Pitt, and A. Scharmann, Radiat. Prot. Dosim. 85, 113 (1999).

[23] R. Ilic, E.S. Kristof, R. Dijanosic, J. Skvarc. and R. Dobnikar, Radiat. Meas. 25, 453 (1995).

[24] F. Castillo, M. Milanese, R. Moroso, and J. Pouzo, J. Phys. D: Appl. Phys. 30, 1499 (1997).

[25] R.B. Gammage and G. Espinosa, Radiat. Meas. 28, 835 (1997). 